[Aus dem Laboratorium

für allgemeine Pathologie und Histologie der K. Universität Pavia.]

(Leitung: Prof. C. Golgi.)

\title{
Über die Morphologie
}

und den Entwicklungszyklus des Parasiten der Tollwut.

(Neuroryetes hydrophobiae Calkins.)

Von

Dr. A. Negri,

Assistenten und Privatdozenten.

(Hierza TaP. XV-XVII.)

Im Jahre 1903 habe ich die Mitteilung gemacht, daß im Nervensystem wutkranker Tiere und zwar in den Nervenzellen - unter bestimmten Bedingungen - beständig eigentümliche Gebilde anzutreffen sind, die ich damals als Parasiten oder eigentlich als Entwicklungsstadien eines als spezifischer Erreger der Wutinfektion wirkenden Protozoons gedeutet habe.

In demselben Jahre habe ich auch auf die Bedeutung hingewiesen, welche die Ermittlung dieser endozellulären Gebilde in praktischer Hinsicht erlangt und schon damals den Wert dieser Untersuchungsmethode zur Stellung einer raschen, sicheren Diagnose der Infektion dartun können.

Die überall angestellten, in einem verbältnismäBig kurzen Zeitraum zu einer beträchtlichen Anzahl angewachsenen Nachprüfungen haben die von mir zuerst gemachten Erfahrungen völlig bestätigt.

Die Spezifizität und die Beständigkeit der in Frage stehenden eigentümlichen Gebilde, ihre Verteilung auf die verschiedenen Teile des Nervensystems und im Inneren der Nervenzellen, die Gestalt, die Größe, die Art und Weise ihres Anftretens, die Zeit dieses letzteren usw., alles dies wurde von seiten einer ganzen Reihe tüchtiger Forscher zum Gegenstand 
der Untersuchung gemacht. Die hierbei erzielten Resultate bestätigen denn auch vollauf die von mir beschriebenen Befunde und sind daher auf dem Gebiete der Wissenschaft zu einer unangefochtenen Stellung gelangt. Andererseits hat. sich die Untersuchung auf "Wutzörperchen" in der Laboratoriumspraxis rasch eingebürgert und hierin einen Fortschritt geschaffen, über den seit lange her es nicht mehr als zulässig erscheint, irgendwelchen Zweifel zu erheben.

Auf die allgemein angenommenen, anerkannten Erfahrungen halte ich es für unnütz, hier näher einzugehen. Artikel und zusammenfassende Abhandlungen sind seitdem $z u$ verschiedenen Zeiten hierüber erschienen, so daB sich gegenwärtig eine neue synthetische Arbeit in dieser Richtung wohl als überflüssig erweisen würde. Und wohl wenig vermöchte ich in der Tat zu Prof. Frosch' sorgfältiger Monographie ${ }^{1}$ noch hinzuzufügen, wo die Ergebnisse der neuen Untersuchungen über die Ätiologie der Tollwut bis zum Jahre 1907 - in bezug auf die soeben erwähnten Fragen in unübertrefflich klarer, präziser, noch jetzt vollständiger Weise zusammengestellt sind.

Ich werde daher jetzt - beim Wiederzurückkommen auf diesen Gegenstand - mich darauf beschränken, einen einzigen Punkt zu berühren; die „Deutung“" dieser Gebilde nämlich, eine hochwichtige Frage, die heute noch Diskussionen und Einschränkungen zu erfahren hat.

Das Studium des Baues der „Wutkörperchen“ mit recht feinen, präzisen Befunden gibt mir die Bestätigung, da $B$ dieselben wohl als einzellige Parasiten anzusehen sind.

Dies auf Grund ihrer feineren Struktur, die im großen und ganzen derjenigen anderer Protozoen entspricht, sowie auch deshalb, weil bei denselben ein nach bestimmten, konstanten Gesetzen sich abwickelnder Evolutionszyklus zu erblicken ist.

Dieses Studium ist allerdings noch kein vollständiges, aber doch bereits ein so weit vorgeschrittenes, da $B$ es meinem Dafürhalten nach schon jetzt dem Gutachten der Sachverständigen unterbreitet werden kann. Ond dies eben beabsichtige ich mit vorliegender Mlitteilung, worin ich trachten werde, jene Kenntnisse, die wir über den Bau des Mikroorganismus besitzen, mit den Tatsachen, die meine weiteren, zahlreichen, geduldvollen Untersuchungen über die feinere Struktur und den Evolutionszyklus festzustellen gestattet haben, lurz in Zusammenhang zu bringen und zu koordinieren. ${ }^{2}$

${ }^{1}$ Frosch, Lyssa. Kolle-Wassermanns Handbuch d.path. Witroorganismen. Ergänzungsband I. S. 626.

Mit tiefgefühltem Danke gedenke ich hier der mir im Mai 1908 von seiten des R. Accademia dei Lincei und des „Elizabeth Thompson Science Fund“ von Boston 
Auf die neuen, von mir in zwei vorläufigen Mitteilungen (1905 und 1907) bereits bekannt gemachten Tatsachen sei hier in ganz besonderer Weise aufmerksam gemacht.

Die große Mehrzahl der endozellulären „Wutkörperchen“ zeigt ein charakteristisches konstantes Aussehen.

Mag auch Gröbe und Gestalt des Parasiten was immer für eine sein, stets ist - unter zweckentsprechenden Bedingungen - in seinem Inneren das Vorhandensein besonderer Gebilde bemerkbar („Innenformationen"), die sich schematisch in zwei Kategorien gruppieren lassen: a) kleine, rundliche, lichtbrechende, b) gröBere, schwächer lichtbrechende, rundliche oder eiförmig bzw. unregelmäBig gestaltete, körnig aussehende Körperchen.

Die „Innenformationen" sind nicht nur an mit verschieden Methoden gefärbten, sondern auch an - ohne irgendwelche Färbung - Schnitten und Zupfpräparaten sichtbar; mit der gröBten Deutlichkeit lassen sich dieselben am frischen Material, in stark verdünnten Essigsäurelösungen, in Wasser oder in physiologischer Kochsalzlösung wahrnehmen.

Letzteres Verfahren gestattet es, festzustellen, daB es sich nicht etwa um künstliche, auf die Einwirkung der Fixierungsflüssigkeiten, oder auf die hierzu angewandten Färbemethoden zu beziehende Produkte handelt, vielmehr liefert es den Beweis, daB die "Innenformationen" der Ausdruck einer wahren Struktur sind.

Die weiter oben angegebene Einteilung dieser „Formationen" ist jedoch eine etwas schematische. Zwischen den größeren liörnig aussehenden, schwächer lichtbrechenden, rundlich, eiförmig oder unregelmäBig gestalteten und den rundlichen, stärker lichtbrechenden, weit kleineren, bestehen allerdings nennenswerte Unterschiede; doch muß ich dazu bemerken, $\mathrm{da} B$ die Untersuchung zahlreicher Parasiten - mitunter auch eines und desselben Parasiten - das Vorhandensein einer ganzen Reihe von „Formationen" ergibt, welche die Übergangsstufen von einer zur anderen Kategorie bilden.

Bezüglich der Zahl und der Gruppierungsweise der „Innenformationen“" in den einzelnen Individuen besteht kein konstantes Gesetz. So sind an einem und demselben Schnitt, in einer und derselben Zelle Parasiten anzutreffen, von denen einige eine - gewöhnlich zentral, zuweilen aber peripher gelegene - groBe „Formation" anfweisen; um diese letztere liegen die kleinen rundlichen "Formationen“, deren Zabl je nach der Größe des Protozoons eine verschiedene ist, im Kreise angeordnet; neben

zuteil gewordenen reichlichen Unterstützung behufs Weiterführung dieser Studien, die wegen der beträchtlichen Anzahl der dabei geopferten Tiere - worunter zahlreiche Kühe - ganz bedeutende Auslagen erfordert haben. 
diesen Parasiten liegen wieder andere, die anstatt einer einzigen zentral gelegenen „großen Formation", deren zwei, drei, vier schwächer entwickelte in verschiedener Lage enthalten; andere schlieBlich sind mit kleinen rundlichen, fast gleichgroBen Körperchen dicht gefüllt. Zwischen diesen bisher beschriebenen Haupttypen recht zahlreiche Übergangsformen, die jede systematische Darstellung unmöglich machen.

Dies ist nun in der großen Mehrzahl der Fälle das Aussehen des Parasiten: eine Beschreibung davon habe ich bereits in meiner ersten Mitteilung gegeben und - unter Hinzufügung mancher Einzelheiten in den darauffolgenden wiederholt und vervollständigt.

Wie ich bereits hervorgehoben, machen die durch den soeben beschriebenen Bau charakterisierten parasitären Formen - wiewohl sie gerade jene sind, die am gewöhnlichsten und leichtesten in den Zellen wutkranker Tiere anzutreffen sind - nur einen Teil der verschiedenen Arten aus, wie sich der Mikroorganismus darstellt.

So finden sich neben diesen Formen auch solche, worin an Stelle der typischen, rundlich bzw. eiförmig gestalteten, gut individualisierten, in verschiedener Weise beisammenliegenden und angeordneten "Innenformationen" ein gleichförmig feinkörniges Aussehen angetroffen wird.

Auf diese neuen Stadien babe ich bereits im Jahre 1905 hingerviesen. Während ich dieselben anfangs bei Rindern vorgefunden, ist es mir später möglich geworden, sie auch bei anderen Säugetieren (1907) nachzuweisen.

Feinkörnig sehen diese Gebilde aus dank den äuBerst zahlreichen winzig kleinen lichtbrechenden Körperchen, die den ganzen Körper des Mikroorganismus dicht ausfüllen. Mitunter gelingt es auch an frischem Material diese Körperchen an ihrer regelmäBigen Gestalt, ihren steifen, scharfen Umrissen und ihrem Gesamtaussehen, das jenem der bisher beschriebenen parasitären Formen entspricht, leicht und mit Sicherheit zu erkennen.

Andere Male wieder ist es durchaus keine leichte Aufgabe, die Parasiten in frischem Zustande auf dieser Entwicklungsstufe zu diagnostizieren, da sie ihre steifen Umrisse eingebüßt haben und zu körnigen, farblosen, zartrandigen Körpern geworden sind, die mit Rücksicht auf die Natur des sie beherbergenden Gewebes sich leicht der Beobachtung entziehen. Doch lassen sich solche Stadien wohl an gefärbten Schnitten zur Anschauung bringen und erkennbar machen.

Auch diese Formen sind nun tatsächlich nur in den Nervenzellen im Zellkörper oder in den Zellfortsätzen anzutreffen und können in keiner Weise als das Produkt einer Zellentartung gedeutet werden. Wie die übrigen können sie eine bedeutende Größe erreichen; je nach ihrem Sitz in der Zelle zeigen sie eine groBe Formverschiedenheit und färben sich nach Mann vorzugsweise rot - mit einem Worte: ihre innerste Struktur 
und die Sehärfe ibrer Umrisse ausgenommen, ist ihr Verhalten ganz ähnlich demjenigen der sonstigen charakteristischen endozellulären Wutkörperchen, mit denen sie durch eine ganze Reihe von Übergangsformen zusarnmenhängen.

Zu meinen früheren Beschreibungen der verschiedenen Art und Weise, wie der Parasit im frischen Zustande oder nach Mann gefärbt in Erscheinung tritt - Beschreibungen, die bereits kontrolliert und als zutreffend erkannt worden, und deren nahezu vollständige Wiedergabe sich hier als notwendig erwiesen hatte - kann ich jetzt nur noch recht wenig hinzufügen.

Ich will nur - da ich dies für einen Punkt halte, der betont zu werden verdient - nochmals hervorheben, daB die Unterscheidung der beiden Kategorien von „Innenformationen“ des Parasiten eine ganz schematische ist und in Wirklichkeit zwischen "groBen" und "kleinen Innenformationen" keine scharfe Grenze liegt.

Wiederholte, sorgfältige Untersuchungen, die ich unter den allerbesten Bedingungen und am passendsten Material - am frischen Material und an den großen Parasiten angestellt, die mit häufig darin enthaltenen recht deutlichen „Innenformationen“ bei Rindern, mitunter auch bei Hunden angetroffen werden - haben mich immer mehr in der Überzeugung bestärkt, daß die Unterscheidungsmerkmale der verschiedenen „Formationen“" nur auf die Durchmesser zu beziehen sind.

Die durchsichtige, farblose, den Eindruck von in die Grundmasse des Parasiten eingegrabenen Vakuolen hervorrufende Substanz, aus der diese Gebilde bestehen, zeigt bei sämtlichen die gleichen Merkmale und Eigenschaften, mögen nun die "Formationen" klein oder hingegen bedeutend entwickelt sein. Bei diesen letzteren machen sich zuweilen selbst im frischen Zustande lichtbrechende Körnchen in versehiedener Anzahl bemerkbar.

Dies vorausgeschickt und mit Rücksicht auf das entweder im frisehen Zustande oder mit Manns Methode bezüglich der Struktur des Parasiten wahrnehmbare, glaube ich, es sei wohl zulässig, folgendes zu behaupten:

Die endozellulären Stadien des Mikroorganismus zeigen sich in der groBen Hehrzahl der Fälle als gut individualisierte Körper mit sehr scharfen, regelmäßigen Umrissen.

Sie bestehen aus einer "Grundmasse", worin - bei jederlei GröBe, Gestalt und Lage des Parasiten - stets durchsichtige, farblose, gewöhnlich rundliche bzw. eiförmige Körperchen anzutreffen sind. 
Diese zuweilen kleine, sämtlich gleich große Körper („Innenformationen") zeigen nun aber andere Male im Inneren des Protozoons verschiedene Dimensionen. In diesem Falle finden sich die gröBeren gewöhnlich im zentralen, die kleineren hin. gegen im peripherischen Teil des Körpers des Mikroorganimus angeordnet.

Neben diesen - im Nervensystem wutkranker Tiere am häufigsten vorkommenden - Formen finden sich noch andere gleichförmig feinkörnige; ein solches Aussehen ist dem Vorhandensein äuBerst zahlreicher, den ganzen Körper des Protozoons ausfüllender lichtbrechender Gebilde zu verdanken. Von diesen letzteren Parasiten zeigen einige noch seharfe, steife Umrisse; andere hingegen sind wegen ihrer Zartheit nur an gefärbten Schnitten zu diagnostizieren.

In den Figg. 1-20, Taf. $X \nabla$ habe ich versucht, einige der unzähligen, rom Protozoon dargebotenen Bilder zu veranschaulichen. So überflüssig es nun auch erscheinen mag, möchte ich darauf aufmerksam machen, da $B$, wiewrohl sich die Abbildungen auf Mikroorganismen im Nervensystem ron Rindern (Ammonshorn) beziehen, in allen übrigen für die Infektion empfängliche Tiere ganz identische Formen anzutreffen sind.

Meiner Ansicht nach geben die Figuren (bei 2250 facher VergröBerung) eine hinreichend genaue Vorstellung von dem Mikroorganismus, wie er sich im frischen Zustande darstellt, und zwar jedenfalls eine der Wahrheit entsprechendere als Fig. 6, Taf. XV meiner ersten Mitteilung, eine Abbildung, die doch in weitem $\mathrm{MaBe}$ reproduziert worden.

Beim Abzeichnen der Umrisse der einzelnen Bilder bin ich bestrebt gewesen, mit der gröBten Gewissenhaftigkeit und der strengsten Genauigkeit vorzugehen. Wenn trotzdem die Figuren den Eindruck erwecken, als ob sie etwas zu stark schematisiert wären, so ist dies auf den Umstand zurückzuführen, daB es mir bei den Zeichnungen nur möglich gewesen, eine einzige Ebene - die mittlere - des Parasitenkörpers genau darzustellen. Allein gerade diese Ebene ist es, wo man bezüglich der Durchmesser der "Innenformationen" - wenn sie nicht alle fast gleich gro $B$ sind, wie dies eben bei den Mikroorganismen der Fig. 15-20 der Fall ist - die gröBte Verschiedenheit antrifft.

Gern hätte ich auch manchen Parasiten mit feinkörniger Struktur abgebildet, wenn ich hierbei nicht auf Schwierigkeiten gestoßen wäre, die sich für die Bilder im frischen Zustande nicht überwinden lieBen.

Ich muB mich daher auf drei riel schwächer vergröBerte Figuren (bei 1000 facher VergröBerung) des bei Kühen vorgefundenen, nach Mann ge- 
färbten Parasiten beschränken; die erste an einem Ammonshornschnitt, die anderen an Schnitten von Hirnrinde. Diese Abbildungen (Fig. 22 und 23 sind meiner Mitteilung von 1905 entnommen) dürften wobl eine Vorstellung soleher Entwicklungsstufen geben, wie sie an den Schnitten und mit Hilfe der angewandten Wethoden sichtbar sind.

Das soeben beschriebene typische Aussehen ist jedoch lediglich der Ausdruck des gröberen Baues des Parasiten, desjenigen nämlich, das bei Anwendung der Färbungsmethoden - die ich als übliche bezeichnen will — oder bei der Untersuchung am frischen Material zur Wahrnehmung gelangt.

Durch andere, geeignetere Forschungsmittel gelingt es aber, neue feinere Eigentümlichkeiten zu enthüllen. Aus der Gesamtheit dieser letzteren läßt sich nun die Organisation und der Lebenszyklus dieses Wesens entrehmen.

Schon im Jahre 1905 hatte ich beim Färben des Parasiten an Schnitten feststellen können, daß sich bei Anwendung verschiedener Hämatoxylinlösungen und geeigneter Modalitäten Körperchen differenzieren lassen, die ich mit Rücksicht auf ihr Aussehen, auf ihre ausgesprochene chemische Affinität zum Kernfarbstoff, auf die Art und Weise ihres Verhaltens usw. als Kerne gedeutet habe.

An den nämlichen Präparaten ist es mir ferner möglich gewesen, verschiedene Gruppierungen der Kernsubstanz festzustellen und daraus den Sehluß zu ziehen, daß sie im Inneren des Protozoons Veränderungen erfäbrt.

Neben diesen bei den gewöhnlichen Formen des Parasiten anzutreffenden Erscheinungen ist es mir gelungen, noch andere in den körnigen Stadien vorkommende anzugeben, in jenen Stadien nämlich, deren Aussehen nach Mannsche Färbung ich durch die Figg. 21, 22, 23, Taf. XV zu veranschaulichen versucht habe.

Bei Färbung dieser Stadien mit Eisenhämatoxylin — und zwar stets an Schnitten - ließen sich dieselben als die Anhäufung recht zahlreicher, intensiv schwarz differenzierter, schwach länglich oder körnig gestalteter Körperchen erkennen, die ich als Sporen - im weiteren Sinne - bzw. als das Endprodult der Teilung des Mikroorganismus gedeutet habe.

Später habe ich das Studium der Struktur und des Zyklus des Protozoons an Schnitten von in Parafin eingebetteten Stücken aufgegeben und mich unter ausschlieBlicher Anwendung von Romanowskys Verfahren mit Ausstrichpräparaten der Nervensubstanz watkranker Tiere befaBt. 
Diese feine, bei der Forschung zahlreicher Protozoen so hilfreich gewesene Untersuchungsmethode, hatte ich bereits seit längerer Zeit in Anwendung gebracht, nur waren die damit erzielten Resultate anfangs keine besonders zufriedenstellenden. ${ }^{1}$

Durch allmähliche Vervollkommnung der Technik gelangte ich zu Befunden, deren Bekanntmachung unter Zuhilfenahme von Mikrophotographien (1907) ich für zweckmäBig erachtete. Leider haben aber letztere bei der Reproduktion viel an Deutlichkeit eingebüBt.

Die mit Hilfe der Romanowskymethode erzielten Resultate hatten nun die zwei wichtigen bereits vorher gemachten Erfahrungen: das Vorhandensein von Teilungsvorgänge aufweisenden Kernteilen (Chromatin), und das Vorhandensein von aus winzig kleinen Körperchen gebildeten Stadien bestätigt: solehe Körperchen erwiesen sich als aus einem von einer hellen Protoplasmahülle umgebenen zentral gelegenen Chromatinkörnchen zusammengesetzt.

Die in dieser letzten Zeitperiode durchgeführten Untersuchungen liefern - wenn auch bezüglich mancher Einzelheiten modifiziert - eine neue umfangreichere, genauere Bestätigung der schon von mir formulierten allgemeinen SchluBfolgerungen.

Bevor ich nun zur Betrachtung der von mir zur Anschauung gebrachten Vorkommnisse übergehe, halte ich es für zweckmäBig, der Technik zu gedenken, die es mir - nunmehr verhältnismäBig leicht und in der Nehrzahl der Fälle - gestattet, das charakteristische, konstante Wiederauftreten derselben zu beobachten.

Wie bereits erwähnt, ist es Romanowskyfärbung - an Ausstrichpräparaten der Nervensubstanz wutkranker Tiere durchgeführt - die ich seit mehreren Jahren hierfür zur Anwendung gebracht. Allerdings ist der Erfolg derselben bei diesem Material nicht immer ein sicherer, doch lassen sich damit ziemlich regelmäBig vorzügliche, weit bessere Resultate erzielen als mit jeder anderen der bisher angewandten üblichen Methoden.

Ein besonderes Eingehen auf das Ausstreichen des Materials dürfte hier um so mehr als überflüssig erscheinen, als verschiedene Autoren diesbezügliche Angaben gemacht haben; doch mag nicht vergessen werden, daB dies eine groBe Wichtigkeit hat.

${ }^{1}$ Inzwischen war die Romanowskyfärbung noch von anderen Forschern (vgl. A. Wessels Williams und M. Murray Lowden. The etiology and diagnosis of hydrophobia. Journal of infections diseases. 1906. Bd. III. Nr. 3) angewandt und empfohlen worden. 
Mag man nun auf was immer für eine Art vorgehen, eines muB doch stets zum Studium der innersten Struktur des Milkroorganismus im Auge behalten werden: die Nervensubstanz soll nämlich in einer gleichförmigen, dünnen Schicht aufgetragen werden, auch wenn dies auf Unkosten der Integrität der Nervenzellen erreichbar ist; zweitens soll graue Nervensubstanz dazu verwendet werden, die einzige, worin die Parasiten anzutreffen sind.

Diese graue Substanz isoliert zu gewinnen, ist gerade nicht schwer, selbst im Ammonshorn nicht, wo doch die gegenseitige Anordnung der Zell- und Faserschichten eine ziemlich komplizierte ist.

Es genügt nämlich, einen ziemlich dicken, durch zwei zueinander parallel geführte Schnitte gewonnenen Frontalabschnitt des Ammonshorns zwischen zwei Objektträger zu bringen und dabei schwach zusammenzudrücken.

Dadurch treten die versehiedenen Schichten deutlich hervor und es ist dann nach Auseinanderbringung der Gläschen nicht schwer, die graue Substanz zu isolieren und hierauf zum Ausstreichen überzugehen.

Es wäre wohl unnütz, sich auf diesen Vorgang näher einzulassen, da ich hierüber nichts Besonderes zu sagen habe.

Das Material habe ich stets - nach manchem Probieren auf die Deckgläser - auf den Objektträger immer ausgestrichen und hierzu die längere Kante eines zweiten Objektträgers benutzt.

Zweckentsprechend ist meines Erachtens ein die ganze Breite des Glases einnehmendes Streichen, weil man dadurch ein weites Beobachtungsfeld bekommt; in dieser Weise bin ich wenigstens stets vorgegangen.

Vorteilhaft ist es auch, wenn das Ausstreichen in einem einzigen Tempo vollzogen wird, doch schadet es nicht, wenn zur Erzielung der erforderlichen GleichmäBigkeit des Streichens dasselbe auch mehrmals wiederholt wird.

Die übriggebliebene, an der Kante des zum Ausstreichen verwendeten Objektträger noch haftende graue Substanz wird zu neuen Präparaten benutzt - solange eine hierzu genügende Menge, derselben noch vorhanden ist.

Zur Fixierung habe ich - wiewohl auch Methylalkohol dazu verwendbar ist - vorzugsweise absoluten Alkohol gebraucht: die Präparate wurden, nachdem sie bei Zimmertemperatur ausgetrocknet, in denselben gebracht und 20 bis 30 Minuten lang darin belassen.

Aus dem Alkohol herausgenommen, mit Fließpapier getrocknet, der Luft bis zur Verdunstung des Alkohols ausgesetzt, können nunmehr die Präparate nach Romanowsky gefärbt werden; von Vorteil ist es, diese Färbung in den ersten Tagen nach der Fixierung vorzunehmen. 
Von den verschiedenen dafür empfohlenen Modalitäten haben sich zwei am besten bewährt: das bekannte Verfahren mit Giemsalösung und das von Laveran angegebene (Eosin-Methylenblau-Silberoxyd nach Borrel). ${ }^{1}$

Beim ersteren hat sich als zweckmäBig erwiesen, die Objektträger mit nach oben gekehrtem Material auf Glasplatten zu legen, und sie sodann mit einer gerade zu ihrer Bedeckung hinreichenden Menge der färbenden Mischung zu übergießen.

Was nun diese letztere anlangt, so balte ich dafür, daß, wenngleich der Zusatz eines Tropfens der Stammlösung für je ein $1^{\mathrm{ccm}}$ destilliertes Wasser genügend ist, es doch vorteilhaft erscheint, das Mengenverhältnis der Giemsalösung etwas zu erhöhen: z. B. 40 bis 42 Tropfen für je $35^{\mathrm{cm}}$ destilliertes Wasser.

Die Mischung muB, bevor man damit die Präparate übergießt, stark erwärmt werden; auch ist es zweckmäBig, dieselbe alle 10 bis 15 Minuten zu erneuern.

Nach 40 bis 50 Minuten - längstens nach einer Stunde - ist das Optimum der Färbung erreicht; die Präparate werden nun unter starkem Wasserstrahl ausgewaschen, mit FlieBpapier sorgfältig abgetrocknet, an der Luft austrocknen gelassen und in Kanadabalsam eingeschlossen.

Bei Anwendung von Borrels Methylenblau habe ich gleichfalls Platten benutzt. In diesen wird nun zunächst die färbende Lösung bereitet, hierauf werden die Präparate in dieselbe gebracht. Die mit dem Material belegte, nach unten gekehrte Fläche darf die Ebene des Gefäßes nicht berühren; dies wird dadurch erzielt, daß man das Präparat an einem Ende durch ein darunter geschobenes Glasplättchen abgehoben hält.

Nach 40 bis 60 Minuten oder noch früher ist die Färbung des Parasiten - ohne dab man hierzu das Färbemittel zu erneuern braucht vollzogen. Hierauf Auswaschen unter starkem Wasserstrahl, Differenzierung einige Minuten lang mittels 5 prozentiger Tanninlösung, sodann Wiederauswaschen, und zwar zuerst unter Wasserstrahl, dann in destilliertem Wasser, Abtrocknen mit FlieBpapier usw.

Wie man sieht, weicht die Färbungstechnik - wofür selbstrerständlich die allerpeinlichste Reinlichkeit der hierbei in Gebrauch stehenden GefäBe unerläBliche Bedingung ist - nur sehr wenig von den klassischen Vorschriften ab.

Wie bereits erwähnt, sind jedoch die damit erzielten Resultate nicht immer in gleicher Weise zufriedenstellende. Der Erfolg ist von einer

${ }^{1}$ Die Methode findet sich angegeben und in ihren Einzelheiten beschrieben bei Laveran und Mesnil, Trypanosomes et Trypanosomiases. Paris 1904. S. 10-11. 
Anzahl Bedingungen abhängig, von denen viele ich wohl kaum mit Bestimmtheit anzugeben wüBte: auch bei stetiger Befolgung der nämlichen Vorschriften kann es vorkommen, daB, während bei einem Tiere fast sämtliche Parasiten gut gefärbt ausfallen, bei den Präparaten eines anderen hingegen die feine Differenzierung vermiBt wird. Daher erscheint es für jedermann, der Nachuntersuchungen anstellen möchte, empfehlenswert, dieselben auf Material verschiedener Herkunft auszudehnen, vor allem aber mit den hierza am besten geeigneten, d. i. den großen Parasiten mit Straßenwut künstiich infizierter, an der natürlichen Evolution der Krankheit zugrunde gegangener Rinder anzufangen.

Es mag schließlich daran erinnert werden, daß die Giemsalösung, die doch mitunter überaus deutliche Bilder geliefert hat, sich - in bezug auf die Beständigkeit der Resultate und namentlich zur Diffenzierung der feineren Stadien des Mikroorganismus - als eine etwas weniger geeignete als die Mischung Eosin-Borrelsches Blau erwiesen. Mit Rücksicht auf diesen Umstand halte ich es für geraten, noch weiter zu empfehlen, nach erfolglos gebliebener Anwendung von Giemsalösung das Material ohne vorherige Heranziehung von Laverans Färbung nicht aufzugeben; ja zweckmäBig ist es immer, sich beider Methoden zu bedienen. Die Präparate aber müssen für jedes Tier in beträchtlicher Anzahl vorhanden sein, sie erfordern zur Auffindung der - mitunter wahrhaftig nicht sehr häufig anzutreffenden - beweisenden Punlite eine sorgfältig und geduldvoll durchgeführte Untersuchung.

Von groBer Wichtigkeit ist bei letzterer Methode die Differenzierung in Tanninlösung: die Präparate müssen durch eine für jeden einzelnen Fall, je nach dem betreffenden Material, der Dauer der Färbung usw., wechselnde Zeit darin liegen . bleiben: diese Zeit läßt sich nun jeweilig nach mancherlei Probieren bestimmen.

Genauere Angaben über die beiden Methoden wüßte ich wohl kaum noch zu liefern: denn, sind nun einmal die in dieser Richtung geltenden Grundsätze bekannt, so ist der Erfolg dieser Methoden zum großen Teil von jener Erfahrung abhängig, die jeder durch wiederholtes, fleiBiges Untersuchen sich selbst aneignen soll.

Bei gehörig vollzogener Romanowskyfärbung zeigt sich die „Grundmasse" des Mikroorganismus in gleichförmiger Weise mehr oder weniger blau gefärbt (Taf. XVI und XVII, Figg. 1 bis 43).

Auf dem blauen Grunde des Parasiten tritt eine mehr oder weniger bedeutende Anzahl Körnchen hervor, welche die rote bzw. violettrote Färbung 
des Chromatins der Protozoen angenommen haben. Neben diesen Körnchen sieht man öfters in der mehr zentral gelegenen Partie des Parasitenkörpers ein oder mehrere rund bzw. eiförmig gestaltete groBe Gebilde; dieselben bestehen aus einer anscheinend homogenen, mehr oder weniger deutlich rosenrot gefärbten Substanz, worin gleichfalls rot bzw. violettrot differenzierbare Körnchen und Klümpchen enthalten sind (Taf. $X V$, Figg. 1 bis $9=11$ bis 21 ).

Offenbar stellen diese groBen Gebilde die gröBeren, im frischen Zustande und mittels anderer Methoden wahrnehmbaren "Innenformationen" dar.

Dagegen entsprechen die kleinen in der "Grundmasse" verteilten, intensiv rot gefärbten Körnchen dem Zentralteil der „kleinen Innenformationen". Ein solches Verhältnis gelangt bei den Ausstrichpräparaten deshalb nicht zur Wahrnehmung, weil die Umrisse der ,kleinen Innenformationen" häufig durch die blaue Färbung der "Grundmasse" verdecht sind, wird aber bei den Parasiten sichtbar, die an Schnitten gefärbt werden, wo die Umrisse der „Formationen“ - selbst der kleineren - ibre deutliche Wahrnehmbarkeit haben. [Mit Manns Methode zuweilen (siehe Taf. V, Fig. 1 meiner ersten Mitteilung 1903) mit den Hämatoxylinlösungen (siehe Figg. 1 bis 10 meiner Mitteilung von 1905) mit Romanowskyfärbung selbst, auf Schnitte angewendet (1907)].

Bei dieser genauen Übereinstimmung zwischen den im frischen $\mathrm{Zu}-$ stande oder mit den gewöhnlichsten Methoden wahrnehmbaren Bildern und den mit Hilfe des Romanowskyschen Verfahrens erhaltenen, wird es leicht begreiflich, wie auch die nach diesem letzteren behandelten Parasiten in bezug auf Größe, Zahl und Gruppierung der "Innenformationen" das verschiedenartigste Aussehen zeigen können.

In dieser Richtung halte ich den Vergleich zwischen den Figuren ron Taf. XV und jenen der nächstfolgenden für hinreichend.

Analog dem, was beim Parasiten im frischen Zustande bezüglich der zwischen den ,größeren und den kleineren Formationen" bestehenden Verhältnisse beobachtet worden, findet sich bei den nach Romanowsky gefärbten Präparaten zwischen den größeren - aus einer rosenroten homogenen Substanz und chromatischen Klümpchen bestehenden Körpern und den kleinen chromatisehen Körnchen, eine ganze Reihe von Übergangsformen, die mitunter auch bei einem und demselben Mikroorganismus anzutreffen sind (Taf. XVI, speziell Figg. 14 bis 21).

Bei den gröBeren „Formationen" lassen sich stets - solange es ihre Durchmesser gestatten - die beiden obenerwähnten Bestandteile differenzieren.

Die eine zuweilen erst schwach angedeutete, andere Male wieder eine etwas deutlichere rosenrote Färbung annehmende Substanz - was nur 
mit der verschiedenen Intensität und Einwirkungsdauer der färbenden Lösung zusammenhängt - weist stets die gleichen Merkmale auf. Die zwischen den einzelnen „Formationen" bestehenden Unterschiede sind bezunglich dieser Substanz und soweit zu ersehen ist, nur auf das Volum dieser letzteren zu beziehen, die auch bei Ausstrichpräparaten in der Regel ihre rundliche bzw. ovale Form beibehält.

Dagegen zeigen die in dieser rosenfarbigen Substanz enthaltenen, intensiv sich färbenden Chromatinklümpchen eine verschiedene GröBe und ein verschiedenartiges Aussehen, die einer besonderen Beschreibung wert erscheinen.

Ich gehe hier nun ohne weiteres zur Darlegung der gemachten Erfahrungen über, wobei ich mich jedoch der Kürze und Klarheit halber auf wenige Angaben über die eigentümlichsten und wichtigsten derselben beschränken will; als Typus wähle ich hierzu den Parasiten, wie er sich bei Rindern darstellt.

Der Mikroorganismus der Tollwut erreicht oft bei den Rindern eine bedeutende GröBe und enthält zuweilen wirklich ansehnliche „Innenformationen".

Eben bei den größeren „Formationen" ist es', wo das eigenartige Verbalten der chromatischen Klümpchen am deutlichsten zutage tritt, wie aus Figg. 1 bis 9 Taf. XVI zu ersehen ist. In diesen Figuren habe ich getrachtet, eine Reihe von Parasiten abzubilden (bei 2250 facher VergröBerung), von denen jeder mit einer "Innenformation" von beträchtlicher Größe versehen ist, so daB mitunter ein guter Teil des Protozoons von diesem Gebilde eingenommen wird.

Dem bereits angegebenen Gesetze entsprechend, zeigt sich bei jedem Parasiten die gröBte "Formation" - die einzige, die ich vorläufig in Betracht ziehen will - aus zwei Bestandteilen zusammengesetzt: einer helleren, sich rosenrot färbenden Substanz und chromatischen Klumpen bzw. Körnchen, die eine intensiv rote Färbung angenommen haben und deren Zahl und GröBe bei den einzelnen Parasiten eine wechselnde ist.

Wie aus den Figuren zu ersehen ist, sind manchmal die chromatischen Klumpen verhältnismäBig gro $B$ und ihre Durchmesser nahezu gleich (Figg. 1 bis 3, Taf. XVI), zuweilen hingegen von der verschiedensten Gröbe (Figg. 4 bis 5 , Taf. XVI).

In den Figg. 6 und 7, Taf. XVI sind die groBen chromatischen Klumpen der vorhergehenden Figuren nicht mehr anzutreffen; anstatt dessen macht sich eine groBe Anzahl von nahezu gleich großen chromatischen Körnchen bemerkbar. Ebenso zahlreich, aber noch kleiner, sind letztere in den Figg. 8 und 9. 
Wāhrend auch bei nicht gar starken VergröBerungen selbst die gröBeren chromatischen Klumpen den Eindruck von homogenen Körperu hervorrufen können, macht man bei Anwendung der stärksten und besten Linsensysteme and bei passender intensiver Belenchtung die Wahrnehmung, daB ein solches Aussehen in Wirklichkeit ihrer feineren Struktur nicht entspricht.

Ich kann hier nur auf die Figg. 1 bis 5, Taf. XVI verweisen, die besser als jede Schilderung eine Vorstellung von den ins Licht stellbaren Vorkommnissen zu geben imstande sind.

Wie man sieht, finden sich in chromatischen Klumpen - wenn die Färbung keine zu starke ist (wie z. B. in Fig. 4) - noch stärker gefārbte Partien in Gestalt von rundlichen Körnchen oder kleinen, länglich geformten Massen. In den größeren Klumpen habe ich in der Regel Körnchen in verschiedener - jedoch niemals gar groBer - Anzahl (Figg. 1 bis 4), in anderen etwas kleineren Klümpchen hingegen häufig zwei nierenförmig gestaltete, zueinander parallel gelagerte, ungefähr an die Anordnung eines Diplococcus erinnernde chromatische Massen wahrgenommen.

Ohne auf weitere Einzelheiten über die Art und Weise einzugehen, wie sich die chromatische Substanz differenziert - was wegen der Kleinheit und Aufeinanderlagerung der Bilder, sowie auch mit Rücksicht darauf, $\mathrm{daB}$ die Färbung nicht immer mit der nämlichen Intensität wirkt, ein überaus schwieriges Studium ausmacht —, will ich hier nur daran erinnern, daB die soeben beschriebenen, in den gröBeren chromatischen Klümpchen deutlich wahrnehmbaren Strukturen sich auch in den kleineren wiederfinden. Bei allen wird es möglich - wenn es nur der Färbungsgrad gestattet - in je nach den Fällen mehr oder weniger deutlicher Weise, die gleichen Anordnungen $z \mathfrak{u}$ beobachten, bis man schlieBlich $\mathrm{zu}$ den kleinen; eben in den Figg. 7, 8, 9 abgebildeten Körnchen gelangt.

Die in ihren Hauptzügen bisher mitgeteilten, mit der gröBten Deutlichkeit und - soweit eine Wertschätzung derselben möglich ist - regelmäBig zu erhebenden Befunde kommen bei sämtlichen "Innenformationen" des Protozoons vor (Figg. 1 bis 20, Taf. XV). Mag ibre Zahl im Inneren des Parasitenkörpers und ihre Größe was immer für eine sein, stets gehen sie aus einer hellen, ihre Form und ihr Gesamtaussehen bedingenden Substanz sowie aus elektiv differenzierten Körperchen hervor. Besitzen letztere eine gewisse Größe, so sind auch an ihnen die oben beschriebenen Vorgänge einer weiteren feineren Differenzierung erkennbar.

GröBere chromatische Klümpchen sind auch bei nicht 'sehr ausgesprochenen „Formationen“ anzutreffen; im allgemeinen hängt aber die Größe der Chromatinkörnchen mit den Durchmessern des Gebildes zu- 
sammen. Daraus ergibt sich, $\mathrm{daB}$ bei jenen Parasiten, wo im frischen Zustande die "Innenformationen" kleine Körperchen von nahezu gleicher Größe sind, bei den gefärbten Präparaten ein gleichmäBig feinkörniges Aussehen $\mathrm{zu}$ finden ist.

Man gelangt nun zu den Figg. 20, 21, Taf. XVI und 22, Taf. XVII. Mit denselben ist aber die Reihe der verschiedenen Gestaltungen des Protozoons noch nicht erschöpft.

Neben den parasitären Formen, worin die chromatische Substanz meistenteils in gleich groBe, im Zentrum bier und da noch zu etwas gröBeren Klümpchen zusammengeballten Körnchen umgewandelt ist (Figg. 20, 21, Taf. XVI und Fig. 22, Taf. XVII), finden sich noch andere, in denen dieselbe sich in Form von kleinen gleichmäBig verteilten Körnchen umhergestreut zeigt.

In diesen Stadien ist es in der Grundmasse des Protozoons, und zwar auf Unkosten derselben, allmählich zur Bildung einer lichtbrechenden, verfärbten, jedes einzelne Chromatinkörnchen umgebenden Ringes gekommen, so daß der ganze Parasit aus der Gesamtheit von dichtgedrängten Körperchen zusammengesetzt erscheint (Figg. 23 bis 31, Taf. XVII).

Manche dieser körnigen Parasiten - die genau den im frischen $\mathrm{Zu}$ stande oder vermittelst anderer Methoden weit weniger deutlich sichtbaren entsprechen - weisen noch regelmäBige, scharfe, deutlich wahrnehmbare Umrisse auf (Figg. 23 bis 27, Taf. XVII); bei anderen hingegen zeigt sich an mancher Stelle der Peripherie die scharfe Abgrenzungslinie unterbrochen.

In diesem Falle wird es mitunter möglich, das Heraustreten winzig kleiner, gleich großer Körperchen zu beobachten. Diese letzteren - zweifellos die nämlichen, in die der Parasit in toto umgewandelt - bestehen aus einem von einer hellen, recht deutlich kontourierten Hülle umgebenen Chromatinkörnchen.

$\mathrm{DaB}$ der durch das Ausstreichen bedingte mechanische Insult die das Heraustreten dieser Körperchen veranlassende Ursache abgibt, ist wohl mehr als wahrscheinlich. Die Sache verliert jedoch gar nichts von ihrer Bedeutung, indem es ja dadurch erwiesen ist, daB in diesem Stadium der Parasit sich aus gut individualisierten, gleich großen Körperchen zusammensetzt, die leicht frei gemacht werden, während man mit aller. Bestimmtheit behaupten darf, daB bei den vorher beschriebenen Gebilden dies niemals der Fall ist.

Die in ihren Einzelheiten aus den Figg. 28 bis 34, Taf. XVII deutlich ersichtliche Erscheinung bedarf wohl kaum einer weiteren Aufklärung. In den Figg. 28 bis 31 ist der Mikroorganismus ein noch nahezu in toto kompakter Körper, in den Figg. 32-34 hingegen ist dessen Zerfall in eine 
Menge neuer, winzig kleiner Individuen ein bei weitem vorgeschrittener und auffalligerer. Bei diesen letzten Bildern, die sich mit absoluter Sicherheit auf den Parasiten der. Tollwut beziehen lassen, halte ich nun mit meiner Beschreibung inne.

Wiewohl ich bisher bedeutend entwickelte Formen des Mikroorganismus in Betracht gezogen, so muB ich doch nun hinzufügen, daB die oben dargelegten Erscheinungen bei allen Parasiten zur Wahrnehmung gelangen, mögen nun letztere was immer für eine GröBe erreicht haben. In dieser Hinsicht dürfte es genügen, auf die Figg. 35 bis 39, Taf. XVII zu verweisen.

Ich halte es schlieBlich nicht für nutzlos, eine Anzahl kleinerer parasitärer Formen gefärbt abzubilden (Figg. 40 bis 42 , Taf. XVII).

Es sind dies die kleinsten noch mit Sicherheit erkennbaren. Im frischen Zustande und bei Anwendung der üblichen Methoden zeigen dieselben eine einzige „Innenformation“, zuweilen zwei, mitunter auch drei von verschiedener Größe.

Durch Romanowsky läBt sich auch bei diesen kleinen Parasiten die Gegenwart von chromatischer Substanz nachweisen, die sich in elektirer Weise differenziert: bei manchen derselben findet sich eine einzige zentral gelegene chromatische Anhäufung (Figg. 40 und 41); wo die bereits erwähnten feineren Differenzierungsvorgänge zur Beobachtung gelangen; bei anderen hingegen zeigt sich neben dem größeren Zentralhaufen ein kleineres Körnchen (Fig. 42). Mit den Durchmessern des Protozoons nimmt auch der chromatische Anteil desselben zu, die chromatischen Klümpchen werden zahlreicher (Fig. 43), so geht es allmählich zu den Bildern der Figg. 35 bis 39 , sowie zu den anderen bereits beschriebenen über.

Bisher die Tatsachen.

Ebenso beständig und regelmäBig wie in den Parasiten des Nervensystems wutkranker Rinder sind diese Tatsachen auch bei den von jedem sonstigen für die Infektion empfänglichen Tiere herstammenden Parasiten anzutreffen.

Bei ihrer Darlegung bin ich stets objektir vorgegangen; auf das $\mathrm{Ab}$ zeichnen der Bilder - deren mikrophotographische Wiedergabe wohl schwerlich in deutlich anschaulicher Weise gelungen wäre - habe ich die allerpeinlichste Sorgfalt und die allergrößte Präzision verwendet, wie dies hervorragende Fachmänner und zahlreiche Forscher anerkannt haben.

Was sich vielleicht an den Figuren aussetzen lieBe, ist das, daB dieselben an Deutlichkeit und Feinheit den Präparaten zurückstehen. Präparate und Figuren sind bereits gröBtenteils zu individuellen bzw. Kollektiv- 
demonstrationen benutzt worden; ich brauche nur an die letzte in der Versammlung der Società Italiana di Patologia zu Palermo im April 1908 von mir gemachte zu erinnern.

Ich bin der Ansicht, daB sämtliche durch meine Untersuchungen ins Licht gestellten Erscheinungen den Beweis liefern, daB man es bei den "Tollwutkörperchen" mit Gebilden zu tun hat, die konstante Merkmale und eine konstante Struktur aufweisen, im Innern der Nervenzelle ein Wachstum erfahren und durch eine Reihe von innersten nach bestimmten, gleichfalls konstanten Gesetzen vor sich gehenden Modifikationen einem Teilungsproze $B$ unterliegen, infolgedessen das ursprüngliche Gebilde in toto in eine bedeutende Anzahl durchwegs gleich großer, die ursprüngliche Struktur des Muttergebildes besitzender Tochterelemente (Sporen) umgewandelt wird, welch letztere sich auch freimachen können.

Die Durchmesser der neuentstandenen Individuen, in die der Mikroorganismus am Ende seines endozellulären Lebens zerfällt, gestatten hier manche Vorkommnisse zu erklären, die anfangs in Dunkel gehüllt, ja sogar der Annahme der parasitären Natur zuwiderlaufend erscheinen mochten.

Ist nun einmal die Existenz von Stadien erwiesen, wo der Parasit in der Form einer Spore eine minimale GröBe und ein solches Aussehen besitzt, daß derselbe, wenn er vereinzelt steht, mit den zu unserer Verfügung stehenden Mitteln sich weder mit Sicherheit identifizieren, noch von der Granula des Gewebes, in das er eingedrungen, differenzieren läßt, so wird es leicht begreiflich, wie es nicht möglich ist, dessen Gegenwart - wenn auch in anderer Gestalt, die aber doch stets eine sehr kleine sein $m u B$ - in jenen Teilen des Nervensystems oder anderer Organe festzustellen, die trotz ihrer Virulenz die typischen endozellulären, parasitären Formen nicht zeigen, und wie ferner solche winzig kleine, noch sehr wenig bekannte Gebilde die porösen Kerzen passieren können.

Es besteht somit zwischen den aus dem Experiment sich ergebenden und den durch, die unmittelbare Beobachtung gewonnenen Resultaten keinerlei Widerspruch, sondern vielmehr ein harmonischer Komplex von Erscheinungen, die immer mehr bestätigen, daB man es mit Körpern zu tun hat, die einen charakteristischen Zyklus aufweisen, der nur einem lebenden Wesen zukommen kann.

Andererseits zeigt das Protozoon die wesentlichen Bestandteile jedes anderen unizellulären Wesens: die "Grundmasse", die wir nun auch als Protoplasma ansprechen können, die "Innenformationen", die sich als Kerne deuten lassen. Bei diesen letzteren sieht man einen sich differenzierenden chromatischen Teil, der alle Merkmale des Chromatins besitzt, eine sehr schwach sich färbende Substanz, die ich mit Rücksicht auf den 
jetzigen Stand unserer Kenntnisse über die Kernstruktur nicht genau zu definieren wüßte.

Bei den Parasiten im frischen Zustande sind es die Kerne, die sich als farblose, helle, vakuolenartige Gebilde darstellen. Und im Inneren der Kerne, speziell im Chromatin ist es, wo die Modifikationen, von denen die verschiedenen Stadien des Evolutionszyllus abhängig sind, vor sich gehen.

Wie sich nun ein solcher Zyklus abwickelt, ist schwer zu bestimmen? ja vielleicht auch zu früh.

Immerhin ist man zur Annahme berechtigt, daB - wie es die Figg. 40 und 41, Taf. XVII dartun - der Kern anfangs ein einheitlicher ist.

Mit der GröBe des Parasiten nimmt auch der Kern zu und zerfällt in - wegen der winzigen Kleinheit der Bilder - bisher noch nicht genau aufgeklärter Weise in Klumpen, die im Körper des Protozoons sich gleichmäBig verteilen. Eine oder mehrere dieser Kernmassen können eine bedeutende bzw. auffälligere Ausbildung erreichen. Infolgedessen zeigt der Parasit anscheinend das verschiedenartigste Aussehen, doch bleibt hierbei der allgemeine Strukturplan stets der nämliche und ein unverānderter.

Kommen nun jene unbekannten Faktoren zur Geltung, welche den Parasiten zur Sporenbildung veranlassen - gleichviel welche GröBe derselbe erreicht hat - so erfährt das Chromatin, das sich entsprechend einem jeden Haufen der helleren Kernsubstanz (wahrscheinlich in der am meisten peripher gelegenen Partie der einzelnen Haufen) in Klümpchen verteilt hat, eine Anzahl nach bestimmten Gesetzen wiederbolt vor sich gehender Teilungsvorgānge, bis sie schlieBlich in toto zu kleinen gleich großen Körnchen reduziert ist. Teilungsprozesse des Chromatins sind obne $Z$ weifel die ron mir Seite $\mathbf{4 3 4}$ beschriebenen und in Figg. 1 bis 9, Taf. XVI veranschaulichten feineren Differenzierungen der Chromatinklümpchen, wenn es mir auch, wegen der angeführten Gründe, nicht möglich gewesen, den Mechanismus eines solchen Prozesses mit aller Genauigkeit festzustellen.

Von diesen Stadien, wo das Cbromatin zu winzig kleinen, teilweise noch in den schwächer gefärbten Klumpen enthaltenen Körnchen verwandelt ist (Figg. 8 und 9, Taf. XVI), geht es sodann zu anderen Entwicklungsstufen des Parasiten über, wo die Chromatinkörnchen im Protoplasma umbergestreut liegen; wie dies zustande kommt, wüBte ich für den Augenblick 
nicht genau anzugeben. Ich begnüge mich damit, auf den in Fig. 10, Taf. XVI abgebildeten Parasiten aufmerksam zu machen, wo man den Eindruck bekommt, als wenn eine groBe in zahlreiche in Teilung begriffene Chromatinklumpen sich auflösende "Formation", ähnlich der in den vorhergehenden Figg. 1 bis 9 abgebildeten, vorhanden wäre.

Wie dem auch sein mag, ist nun einmal die Teiluing des ganzen Chromatins - oder nahezu des ganzen - in Form von winzig kleinen gleichförmigen Körnchen erfolgt, das Protoplasma teilt sich um ein jedes derselben herum. Der Mikro. organismus verwandelt sich nämlich in einen Haufen sehr kleiner Körperchen (Sporen), von denen jedes als ein von einer deutlich individualisierten, aus einer hellen, wenig färbbaren, anscheinend homogenen Substanz bestehenden Kapsel oder Hülle umgebenes Chromatinkörnchen sich darstellt.

Die Sporen sind anfangs $z u$ einer einzigen kompakten Masse vereinigt; ihre Zusammenfügung wird dann allmählich eine mehr lockere; auch können dieselben unter Umständen sich voneinander entfernen und zu selbständigen neuen Wesen werden.

Zugunsten dieser allgemeinen Auffassung des Entwicklungskreises des Parasiten - jenes einzigen Teiles, deren Verfolgung möglich ist und sich im Innern der Nervenzelle abspielt - sprechen die von mir erhobenen und veranschaulichten Befunde, sowie auch die Ảhnlichkeit mit den bei anderen Protozoen gemachten Erfahrungen.

$\mathrm{DaB}$ noch anderen Protozoen die soeben beschriebene Kernstruktur zukommt, und daB ferner unter Umständen ein ,zerstreuter Kern ${ }^{61}$ möglich ist - ein Zustand des Kernes, welcher in dem der Sporenbildung vorangehenden Stadium häufig besonders scharf ausgeprägt ist - brauche ich wohl kaum des Näheren auszuführen.

Ebenso weicht der Mechanismus der Sporenbildung von dem bei anderen Protozoen anzutreffenden in keiner Weise $a b$.

\footnotetext{
${ }^{1}$ Calkins bezeichnet diesen Zustand des Kerns beim Parasiten der Tollwut als "Chromidium“.

DaB bei diesem Protozoon das Aussehen des Kerns dem „chromidialen Zustand“ im Sinne Hertwigs entspricht, hat viel Wahrscheinlichkeit für sich. Auf den Gebrauch dieser Benennung habe ich aber verzichtet, um eventuellen MiBverständnissen vorzubeugen, da der Begriff "Chromidium" von seiten mehrerer Autoren sehr erweitert worden, indem man unter demselben eine ganze Reihe von endozellulären Gebilden zusammengefabt hat, die vielleicht voneinander auch verschieden sein könnten.
} 
Sicherlich bietet dieser Mikroorganismus trotz zahlreicher Berührungspunkte mit anderen stets etwas Eigentümliches dar.

Doch glaube ich wohl kaum, dies könne dazu benutzt werden, um die parasitäre Natur desselben in Abrede zu stellen; die gemachten Feststellungen liefern ja den Beweis dafür, daB er bezüglich seiner Struktur und seines Lebenszyklus den für die einzelligen Wesen geltenden Grundgesetzen unterliegt.

Nicht nutzlos ist es, daran zu erinnern, daB man zu diesem SchluB noch durch eine zweite ganze Reihe hochwichtiger - wenn auch indirekter - Argumente gedrängt wird.

Letztere haben ihre sichere Stätze in jener anatomischen Grundlage, die zu derartigen Forschungen unumgänglich notwendig ist, und von manchem Forscher viel zu wenig beachtet wurde.

Daher die Möglichkeit, vom Parasiten der Tollwut Deutungen zu geben, die weder in den Lehren der Mikrobiologie, noch in den durch das fleißige Studium der Zelle, speziell der Nervenzelle, im normalen bzw. pathologischen Zustande gewonnene Kenntnisse irgendwelche Stütze finden.

Diesen rein hypothetischen Deutungen stellte ich nun die zahlreiche Reihe der Tatsachen entgegen, die ich, wie ich bereits zu Anfang der vorliegenden Mitteilung bemerkt habe, dem Urteil der Fachgelehrten unterbreite.

Für diesen Mikroorganismus, den ich in meiner ersten Mitteilung zu den Sporozoen gezählt habe - eine systematische Stellung, die ich dem Parasiten noch immer zuweisen zu können glaube - hat Calkins den Namen Neuroryctes hydrophobiae angewandt, der mit Rücksicht auf die Gesetze der zoologischen Nomenklatur auch beibehalten werden muB. 
Diese Abhandlung wurde der R. Accademia dei Lincei in der Sitzung vom 7. Februar 1909 vorgelegt; über dieselben haben die Herren Mitglieder Prof. Camillo Golgi und Prof. Battista Grassi folgenden Bericht, den ich hier wörtlich übersetzt wiedergebe, erstattet:

„Wohlbekannt ist die von Dr. Adelchi Negri gemachte Entdeckung jener Gebilde, die nach ihm benannt worden und für die Tollwut spezifische sind. Schon gleich zu Anfang war Negri der Meinung gewesen, man habe es mit Protozoenparasiten zu tun, welche den von so vielen Forschern lange und vergeblich gesuchten Erreger dieser Infektion darstellen.

AuBer auf die Merkmale der fraglichen Gebilde stützte Negri seine Annahme auch noch auf die gründliche Kenntnis der Nervenzellen, unter normalen bzw. pathologischen Verhältnissen, die ihn dazu berechtigten, auszuschließen, daß man eventuelle Zellentartungsprodukte vor sich habe. Der Ansicht Negris wurde jedoch die allgemeine Beistimmung nicht zuteil, da bei dem vermeintlichen Parasiten jederlei Merkmale vermiBt wurden, die geeignet gewesen wären, denselben mit Bestimmtheit als lebendes Wesen zu deuten, und auch weil dessen Entwicklungszyklus nicht zu ersehen war.

Ein großes Verdienst Negris ist es, daB er seine Untersuchungen fünf Jahre lang mit Beharrlichkeit fortgeführt. Langwierige, geduldvolle Beobachtungen mit den stärksten und besten Vergrößerungen an mit sehr feinen Methoden gefärbten Präparaten angestellt, haben ihm die Möglichkeit gewährt, eine Reihe von Erscheinungen ins Licht zu stellen, die den Beweis liefern, daß seine ursprüngliche Annahme eine wohl begründete gewesen, d. h. dab die in Rede stehenden Gebilde Parasiten, für Tollwut spezifische, und zwar Protozoen sind.

So gelangt nun Negri durch äuBerst vorsichtiges Zusammenstellen der von ihm beobachteten Stadien zu dem Schlusse, daB dieser Mikroorganismus wächst, und sich durch eine Art von Sporenbildung oder Conitomie nach voraufgegangener komplizierter, vielförmiger Teilung des Chromatins reproduziert.

Allerdings stellt dieser Zyklus - wie dies der Verfasser selbst hervorhebt - keineswegs den ganzen Lebenskreis des Parasiten dar, sondern erst nur einen bemerkenswerten Teil desselben, der viele die Tollwutinfektion betreffende Erscheinungen erklärt.

Wir haben Negris Präparate in dem MaBe, als seine Untersuchungen weiter schritten, wiederholt und aufmerksam besehen, und können die Versicherung geben, daB seine Figuren und Beschreibungen das Wahrnehmbare ganz getreu wiedergeben. 
Mit Rücksicht darauf, daß die „Negrischen Körperchen“ beim gegenwärtigen Stand der histo-pathologischen Kenntnisse sich in keiner Weise als Zellenentartungsprodukte deuten lassen, und daB andererseits die neuen vom Entdecker ins Licht gestellten, die feinere Struktur und die Entwicklungsstufen dieser Körperchen betreffenden Erfahrungen sämtlich entschieden zugunsten der parasitären Natur dieser eigentümlichen endozellulären Körper der Tollwatinfektion sprechen, beantragen die Unterzeichneten die Veröffentlichung der Abhandlungen Negris, in der mit Klarheit und Ordnung das bisher von uns kurz Berichtete ausführlich besprochen wird.".

\section{Literatur-Verzeichnis.}

Mitteilungen des Verfassers über die Ätiologie der Tollwut:

1903. Beitrag zum Studiun der Ätiologie der Tollwut. Diese Zeitschrift. Bd. XIIII. 1903. Zur Ätiologie der Tollwut. Die Diagnose der Tollwut auf Grund der neuen Befunde. Ebenda. Bd. XLIV.

1904. Sull' eziologia della rabbia. La dimostrazione del parassita specifico nell' in fezione rabica degli uccelli. Bollettino della Società Med. Chirurg. di Pavia.

1904. I risultati della nuove ricerche sull' eziologia della rabbia. Lo Sperimentale. anno LVIII.

1905. Sull' eziologia della rabbia. Note sulla morfologia e sul ciclo evolutivo del parassita specifico. Bollettino della Società Med. Chirurg: di Pavia.

1907. Sulla morfologia e sul ciclo evolutivo del parassita della rabbia. Rendicontí della $R$. Accademia dei lincei. Vol. XVI. 1. Sem. Serie 5a. Fasc. 9.

1908. Sulla morfologia e sul ciclo evolutivo del parassita della rabbia. Atti della $D$ Riunione della Società Itátiana di Patologia. Palermo. 


\section{Erklärung der Abbildnngen. \\ (Taf. $\mathbf{X V}-\mathbf{X V I I . )}$ \\ Tafel XV.}

Fig. 1 bis 20. Parasiten in frischem Zustande. Aus dem Ammonshorn von nach künstlicher Infizierung gestorbenen Kühen. Ob. apocr. Zeiss $2^{\mathrm{mm}}$ homog. Immersion, Apert. 1.40, Comp. Ok. 18, Tabusl. $160^{\mathrm{mm}}$.

Fig. 21. Ein Parasit in einer Nervenzelle. Aus einem Schnitt des Ammons. horns einer infolge Bisses eines wütenden Hundes gestorbenen Kuh. Färbung nach Mann. Ob. apocr. Zeiss $2^{\mathrm{mm}}$ homog. Immers., Apert. 1-40, Comp. Ok. 18, Tubnsl. $160^{\mathrm{mm}}$.

Fig. 22, 23. Parasiten in Nervenzellen. Aus Schnitten der Hirnrinde einer an experimenteller Wut zugrande gegangenen Kah. Färbung nach Mann. Ob. apocr. Zeiss $2^{\mathrm{mm}}$ homog. Immers., Apert. 1.40, Comp Ok. 8, Tubusl. $160 \mathrm{~mm}$.

\section{Tafel XVI.}

Fig. 1 bis 21. Parasiten aus Ausstrichpräparaten der grauen Substanz des Ammonshorns von Kühen (experimentelle Wat). Romanowskyfärbung; mit Giemsas Lösung bei den Parasiten der Fig. 1, 2, 3, 4, 5, 6, 7, 8, 9, 11, 12, 13, 16, 17, mit Eosin-Borrelschem Blan bei den Parasiten der Fig. 10, 14, 15, 18, 19, 20, 21. Ob. apocr. Zeiss $2^{\mathrm{mm}}$ homog. Immers., Apert. 1.40, Comp. Ok. 18, Tubusl. $160^{\mathrm{mm}}$.

\section{Tafel XVII.}

Fig. 22 bis 43. Parasiten ans Ausstrichpräparaten der grauen Substanz des Ammonshorns von Kühen (experimentelle Wut). Romanowskyfärbong; mit Giemsas Lösung bei den Parasiten der Fig. 23, 35, 40, 41, 42, 43, mit Eosin-Borrel schem Blau bei den übrigen. Ob. apocr. Zeiss $2^{\mathrm{mm}}$ homog. Immers., Apert. 1.40, Comp. Ok. 18, Tubssl. $160^{\mathrm{mm}}$.

Sämtliche Figuren der drei Tafeln wurden abgezeichnet mit Hilfe der Camera lucida von Apáthy: Blatt in der Höhe des Präparates. 


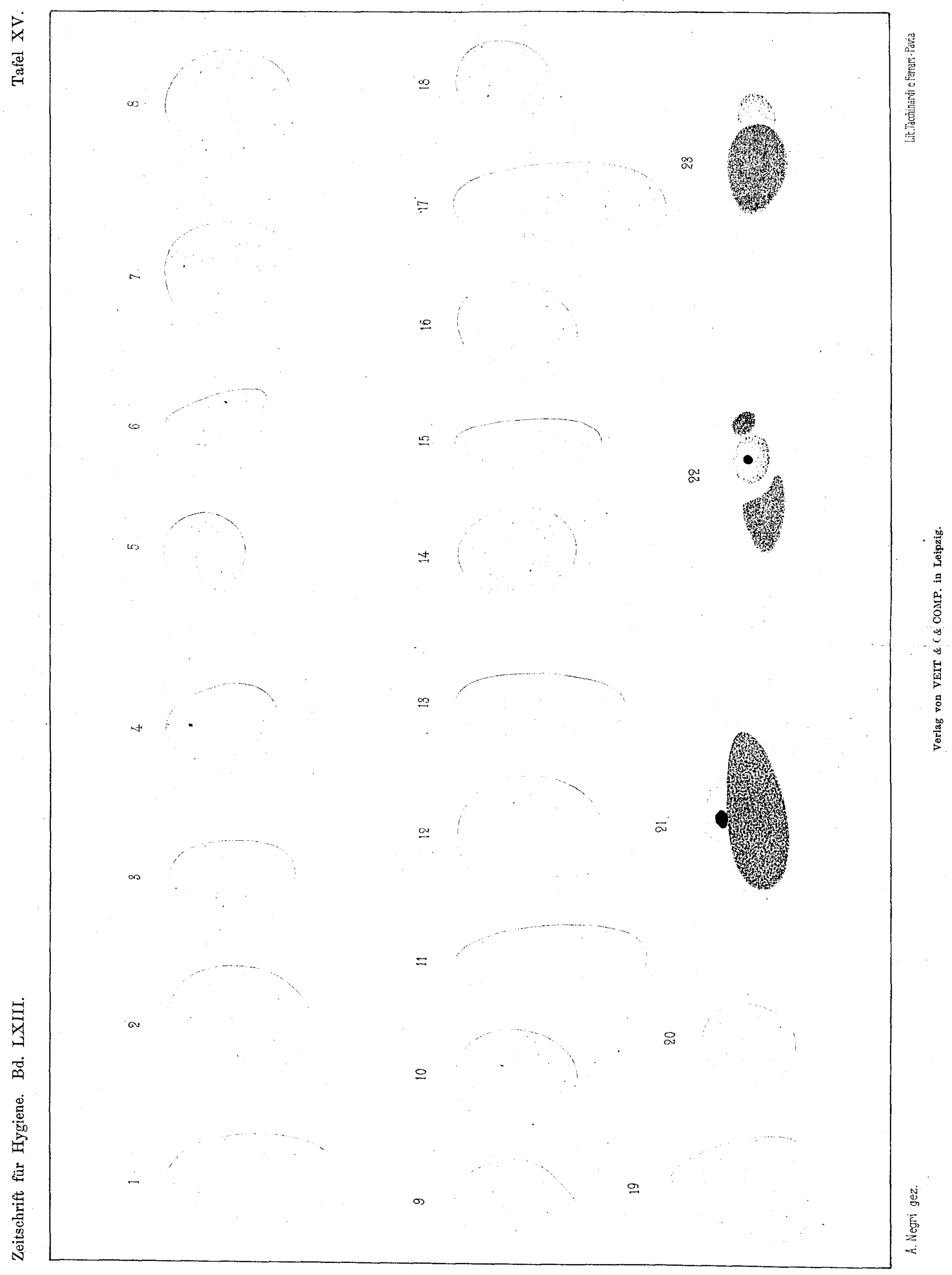




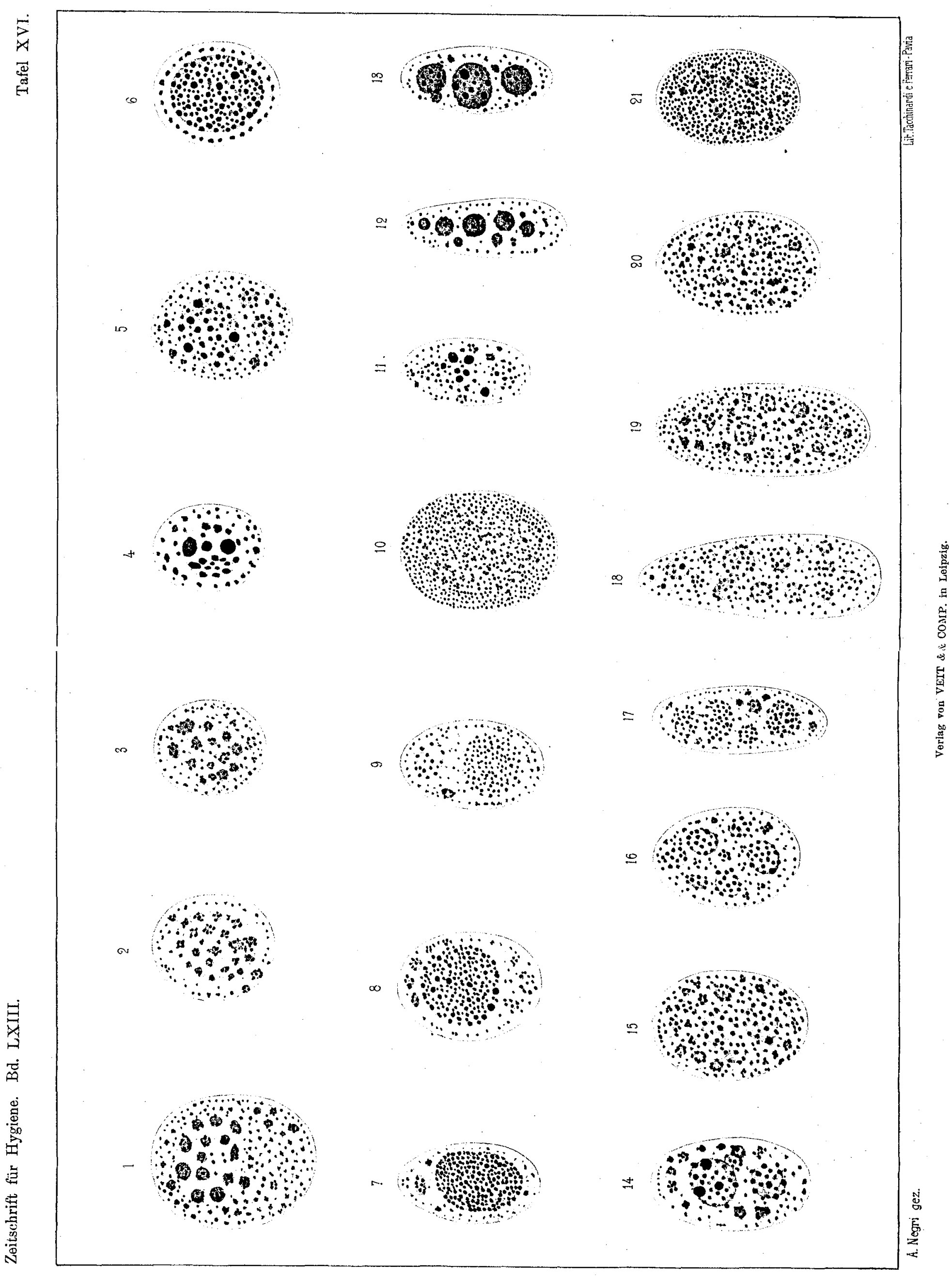


s

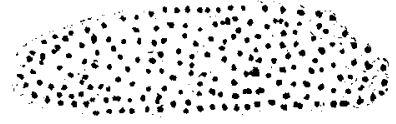

$\stackrel{8}{\circ}$

4

品

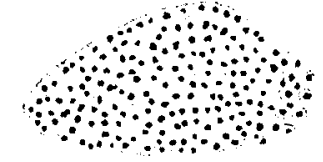

光

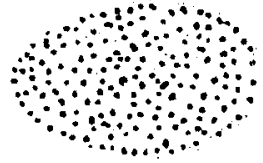

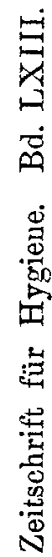

\%

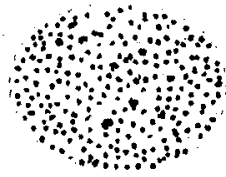

๙

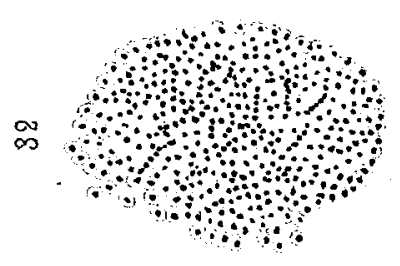

o

$\infty$

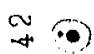

$-\infty$

or

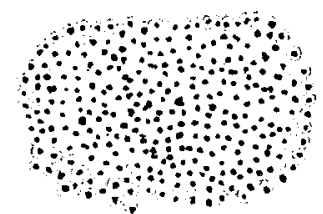

$\infty$

$$
\mp
$$

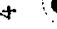

용
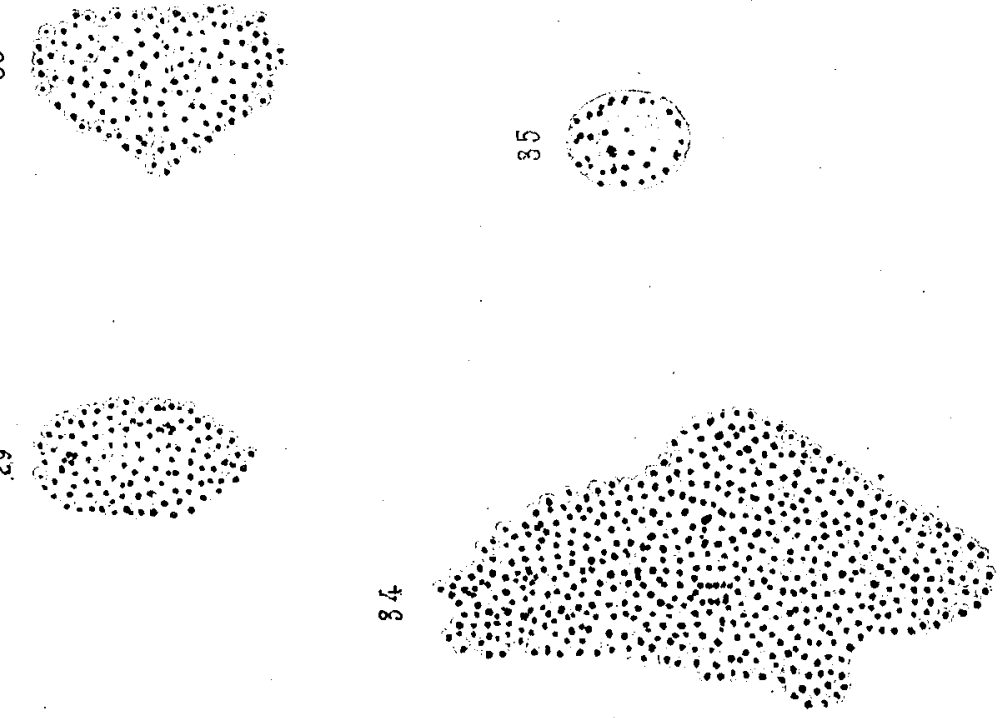

용
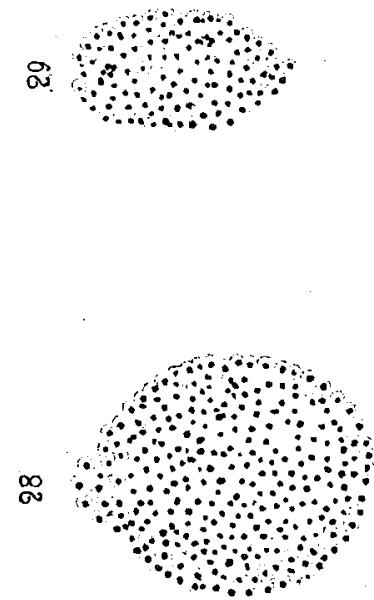

$\infty$

$\infty$ ado 